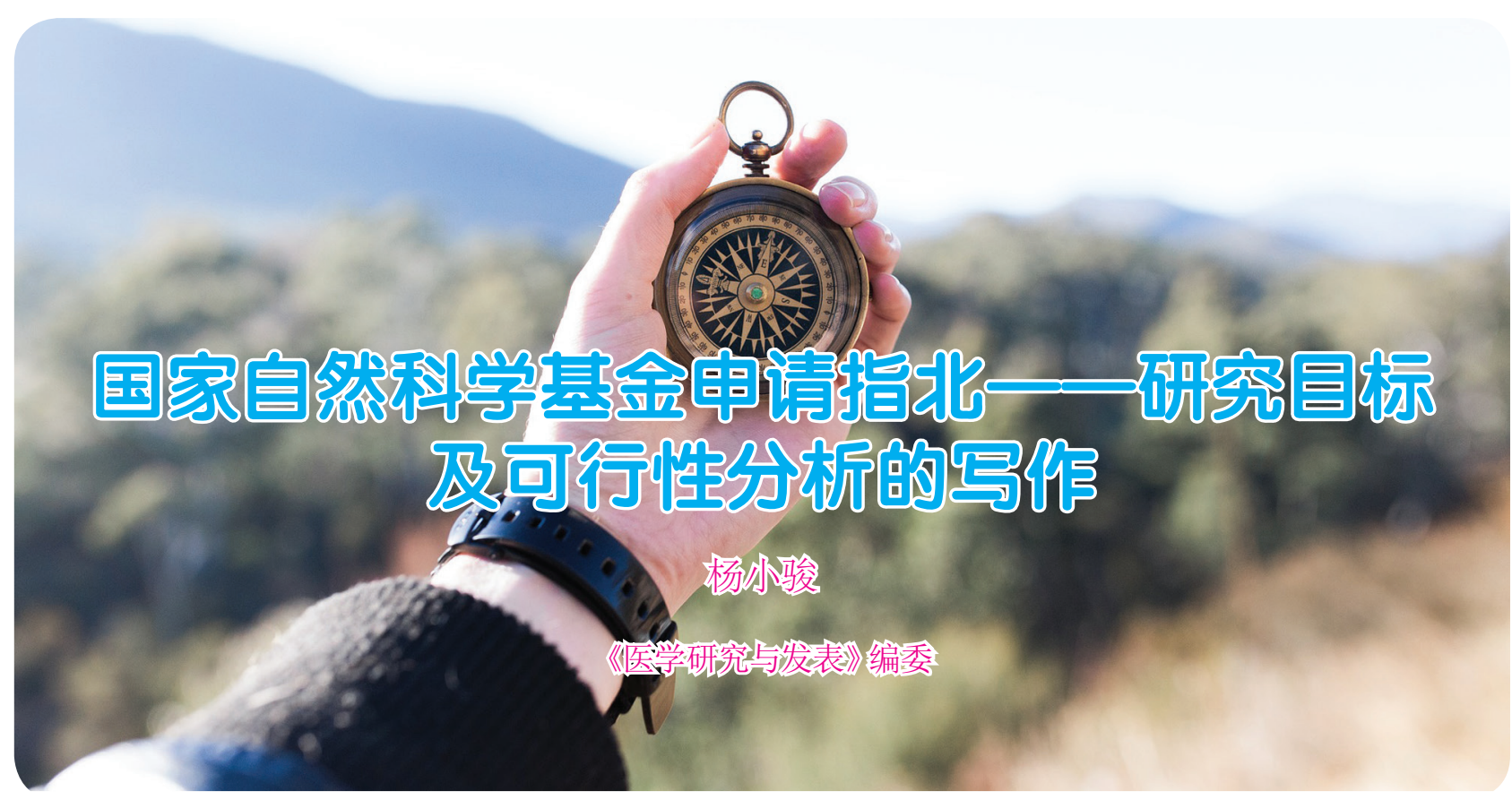

研究目标和可行性分析虽然 字数不多, 但是地位非常关键。 根据立项依据和研究内容最终凝 练出的研究目标一定要明确要点, 研究问题的提法要准确、恰当, 要覆盖到所有的拟解决的科学问 题, 内容要详尽, 但是字数不宜 过多。因此, 对于研究目标需要 字煁句酌, 最好能用最短的篇幅 突出最关键的问题, 并且体现出 研究的难度。在这一部分中, 不 宜写得过于具体, 一来增加了字 数, 二来容易留下漏洞。

可行性分析这一部分可以充 分显示出申请者对于整个课题的 把握能力, 对于评审专家来说, 看完了这一部分基本就确定该项 目是否可以顺利完成。其中可行 性分析主要包括以下几个部分:

1. 是否具有成熟的理论基础: 创新点的提出和解决是否在 理论上可行, 在这一部分中, 建议引用一些高水平的文献以
表明标书的理论具有可行性。 但是切记过犹不及, 要是都 被人做完了, 那么标书也没有 批准的意义了。

2. 研究目标在现有的技术条件 下的可实现性: 在这一部分 中, 要充分展示申请人的学 术背景, 必须要强调所有的 技术都是成熟的，以表明课 题在技术上是没有问题的。

3. 本单位现有设备、实验材料 的完备性: 基本上这个大家 都没有什么问题，现在国内高 校和研究所的硬件设施都已 经赶英超美了。

4. 课题组成员完成课题的能力: 简要来说就是表明所有课题 组成员都有完成相关实验的 能力, 特别是需要强调申请者 本人具有相当强的学术背景。 当然, 如果研究所有大佬坐 镇的话, 可以适当的提一提, 但是度要把握好, 说多了评审
专家会觉得申请人比较无能, 说少了又没什么用。

\section{以本人标书为例 :}

本项目组拥有基础扎实、配 合默契的科研团队，研究队伍 由从事 $\mathrm{XXX} 、 \mathrm{XXX} 、 \mathrm{XXX}$ 及 $\mathrm{XXX}$ 的科研人员组成, 成员均有 参与国家级课题的研究经历, 经 过了严格的科研训练, 熟悉本项 目所涉及的全部技术、方法，为 课题的设计和开展提供了多层次 的支持。在研究所主任 XXX 教 授领导下的团队长期从事 $\mathrm{XXX}$ 的机制研究, 首次发现 XXX 的 生物学功能, 取得了一系列有价 值的研究成果。课题申请人近年 来围绕 XXX 开展研究工作, 对 $\mathrm{XXX}$ 特别是 $\mathrm{XXX}$ 有较深的了解, 近年来以第一作者和通讯作者在 XXX 等 SCI 期刊发表多篇论著, 相关工作积累可为本项目的顺利 完成提供坚实基础。 